\title{
A Pulsed Neutron Dose Monitor
}

\author{
Alfred Klett, Member, IEEE, Albrecht Leuschner
}

\begin{abstract}
A new neutron dose monitor for the measurement of pulsed neutron fields was designed and tested. The concept is based on high energy neutrons activating on ${ }^{12} \mathrm{C}$ target nuclei in the detector materials short-lived radioactive nuclides. The new system comprises two different detectors to measure simultaneously neutrons and charged particles from the decay of instable intermediate nuclear states: a ${ }^{3} \mathrm{He}$ proportional counter tube in a moderator and a plastic scintillator with a photomultiplier. The decay products are detected in timeresolved readout. Analysis of the time distributions provides dose information free of dead time.
\end{abstract}

\section{INTRODUCTION}

$I^{2}$ $\mathrm{N}$ accelerator environments there are usually very special

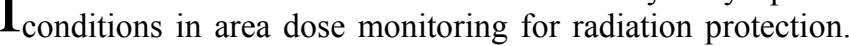
The typical characteristics at accelerators are pulsed radiation fields and high or even extremely high energies of the particles. It is well-known, that many conventional instruments for the detection of ionizing radiation strongly suffer from dead-time effects and have therefore severe limitations in the measurement of pulsed radiation fields [1] In addition many types of detectors have not enough efficiency at high energies and tend to underestimate those radiation field intensities. Even calibration at high energies is an issue, because there are not so many calibration fields available. An overview of these aspects is for instance discussed in reference [2].

Berthold Technologies a German manufacturer of radiation protection instrumentation and Deutsches ElektronenSynchrotron DESY in Hamburg have established a cooperation to address these two problems with high energies and pulsed neutron radiation fields in neutron area dose monitoring. The objective of this joint effort is the design of a completely new system, which should be appropriate for routine operation in accelerator laboratories.

The new approach is resting upon a proposal of DESY. The concept is based on the idea of storing pulsed dose information in an excited nuclear state with a short half-life. Decay products of this intermediate short lived state are being detected in time resolved readout. These events are not subject to dead-time and the threshold neutron energy of the reaction ensures measurement of high-energy neutrons.

Within a cooperation between DESY and Berthold Technologies the new detector system LB 6419 for the measurement of pulsed neutron dose rate was newly designed and it will be soon commercially available.

\footnotetext{
Manuscript received November 21, 2007.

Alfred Klett is with BERTHOLD TECHNOLOGIES GmbH \& Co. KG, 75323 Bad Wildbad, Germany, e-mail: Alfred.Klett@Berthold.com). Albrecht Leuschner is with Deutsches Elektronen-Synchrotron DESY, 22607 Hamburg, Germany
}

\section{The PRINCIPLE OF DETECTION}

High-energy neutrons are hitting upon ${ }^{12} \mathrm{C}$ target nuclei of the detector materials and are generating short-lived ${ }^{9} \mathrm{Li},{ }^{8} \mathrm{Li}$ and ${ }^{12} \mathrm{~B}$ nuclei in the following nuclear reactions:

$\begin{array}{llr}\begin{array}{l}\text { Nuclear } \\ \text { Reaction }\end{array} & \begin{array}{l}\text { Decay } \\ \text { Pattern }\end{array} & \text { Half-Live } \\ { }^{12} \mathrm{C}(\mathrm{n}, \mathrm{X}){ }^{9} \mathrm{Li} & { }^{9} \mathrm{Li} \Rightarrow{ }^{9} \mathrm{Be}^{*}+\beta^{-}+\mathrm{v} & 178 \mathrm{~ms} \\ & { }^{9} \mathrm{Be} * \alpha \alpha+\alpha+\mathrm{n} & \\ { }^{12} \mathrm{C}(\mathrm{n}, \mathrm{p}){ }^{12} \mathrm{~B} & { }^{12} \mathrm{~B} \Rightarrow{ }^{12} \mathrm{C}+\beta^{-}+\mathrm{v} & 20 \mathrm{~ms} \\ { }^{12} \mathrm{C}(\mathrm{n}, \mathrm{p} \alpha){ }^{8} \mathrm{Li} & { }^{8} \mathrm{Li} \Rightarrow \alpha+\alpha+\beta^{-}+\mathrm{v} & 840 \mathrm{~ms}\end{array}$

Neutrons emitted from nuclear reaction (1) are detected in the neutron channel of the system. Charged particles from nuclear reactions (1), (2) and (3) are detected in the plastic scintillator as charged particle channel.

\section{THE DETECTOR SYSTEM}

The detector system comprises a cylindrical moderator made out of polyethylene with diameter $\varnothing 286 \mathrm{~mm}$. The top of the moderator is cone-shaped. In the centre of the moderator there is a ${ }^{3} \mathrm{He}$ proportional counter tube with a diameter of $\varnothing 40 \mathrm{~mm}$. In the moderator there is a Cd-absorber to shape the energy dependent response. At the top of the moderator a plastic scintillator sticks out. The photomultiplier is in the moderator. The cylindrical plastic scintillator has a diameter of $\varnothing 48 \mathrm{~mm}$ and a length of $48 \mathrm{~mm}$. The electronics for both measuring channels is in a steel housing on the bottom side. The overall length of the whole system is about $600 \mathrm{~mm}$. The decay products are detected in time-resolved readout. Analysis of the time distributions provides dose information free of dead time. The data acquisition was done with a STRUCK flash ADC system. It autosynchronizes with the beam pulses by pile-up burst detection and provides time-resolved measurement in both detection channels.

\section{MEASUREMENTS AND ANALYSIS}

The detector was irradiated with pulsed neutron fields at DESY in Hamburg in June 2007. The fields were generated by a $7.5 \mathrm{GeV}$ proton beam in the transfer tunnel from the DESY III synchrotron to the PETRA storage ring. By switching of a bending magnet in the injection line a complete beam loss was generated in the shielding. It consisted out of the concrete walls of the tunnel and the hall and the soil in between and it had about $2.5 \mathrm{~m}$ of total thickness. Behind the shielding there was the irradiation platform inside the PETRA experimental hall. The proton beam had a repetition rate of 
$0.25 \mathrm{~Hz}$ with a microstructure of 10 bunches spaced at $96 \mathrm{~ns}$ length. The total width of one pulse train was almost $1 \mu$ s. On the irradiation platform the neutron energy spectrum is expected to be in equilibrium for normal concrete as shown in Fig. 3. [3], [4]

The doses were monitored with several independent passive dose meters, which are commonly used at DESY. A single bunch train generated a neutron dose of $50 \mu \mathrm{Sv}$ at the neutron probe's position in the experimental hall. From earlier measurements it was known, that neutrons with energies below $20 \mathrm{MeV}$ contribute with $27 \mu \mathrm{Sv}$ while high energy neutrons contribute with $23 \mu \mathrm{Sv}$ to the total neutron dose. The gamma dose was $7 \mu \mathrm{Sv}$ per bunch train.

The detector signals from the neutron channel and from the charged particle channel were recorded with their respective time-stamps. For analysis these events were accumulated in time-spectra. The background was estimated at large delays of a few seconds and was subtracted in the spectra. The time spectra were then rebound in logarithmically equidistant intervals in a lethargy type of representation. A simple fit function with one or two exponentials was used to model the data. Fig. 1 displays the two peaks of the decay products from the ${ }^{8} \mathrm{Li}$ and ${ }^{12} \mathrm{~B}$ nuclei in the scintillation channel. Fig. 2 displays the peak of the decay neutrons from the ${ }^{9} \mathrm{Li}$ nucleus.

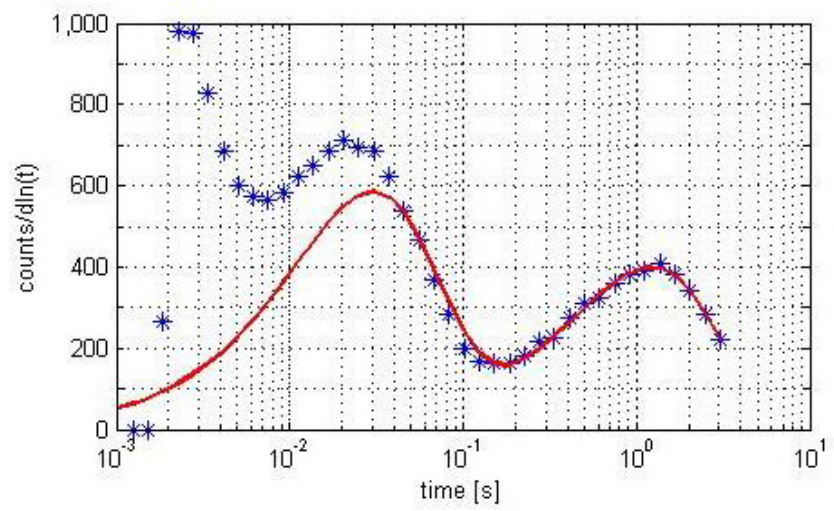

Fig. 1. Delay time distribution of scintillation counter channel

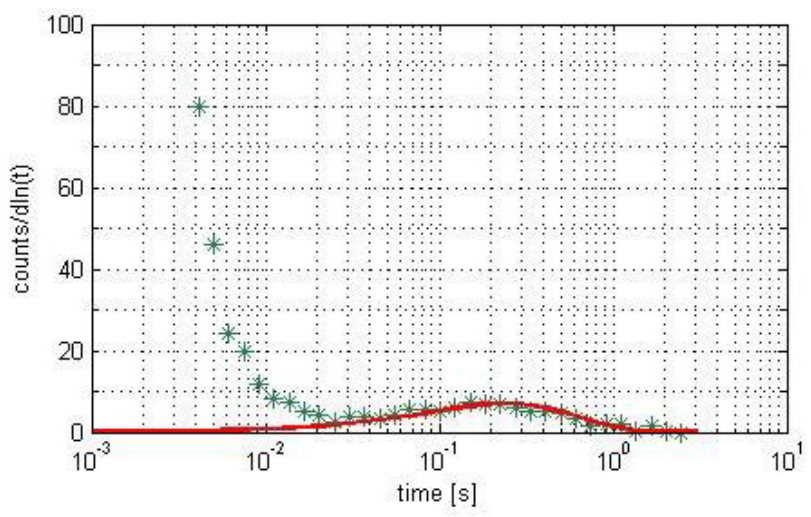

Fig. 2. Delay time distribution of ${ }^{3} \mathrm{He}$ neutron channel

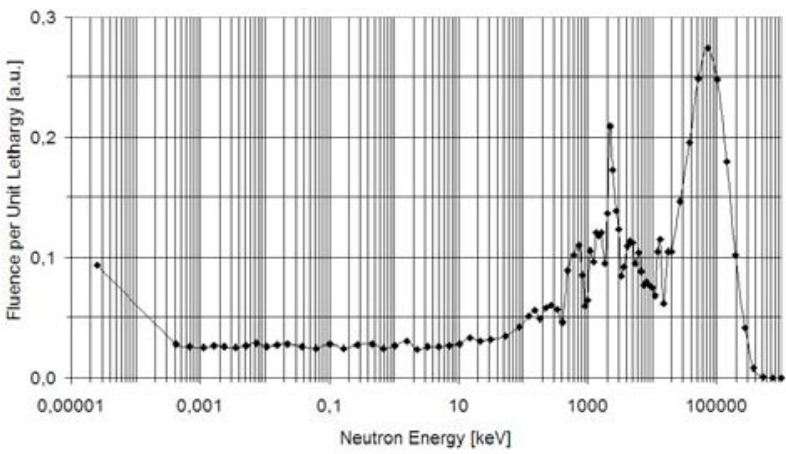

Fig. 3. Neutron energy spectrum in lethargy representation

TABLE I

NEUTRON DOSE RESPONSE

\begin{tabular}{|c|c|c|c|}
\hline Nucleus & $\begin{array}{l}\text { Noise } \\
\text { Counts }\end{array}$ & $\begin{array}{c}\text { Net } \\
\text { Counts }\end{array}$ & $\begin{array}{c}\text { Counts/Dose } \\
{\left[\mathbf{\mu S v}^{-1} \mathbf{]}\right.}\end{array}$ \\
\hline${ }^{12} \mathrm{~B}$ & 372 & 1520 & 30 \\
${ }^{8} \mathrm{Li}$ & 372 & 1086 & 22 \\
${ }^{9} \mathrm{Li}$ & 4.1 & 19.0 & 0.38 \\
\hline
\end{tabular}

\section{CONCLUSIONS}

The new approach with time-resolved readout for the measurement of pulsed high-energy neutron doses was successfully tested. The decay products emitted by the intermediate radioactive nuclei ${ }^{8} \mathrm{Li},{ }^{9} \mathrm{Li}$ and ${ }^{12} \mathrm{~B}$ were easily identified and analyzed in the recorded delay time spectra. The responses to neutron dose are reported in Table I.

\section{REFERENCES}

[1] Glenn F. Knoll, Radiation Detection and Measurement, 3rd Edition John Wiley \& Sons, Inc., New York, 1999

[2] P. Bilski, J. Blomgren, F. d'Errico, A. Esposito, G. Fehrenbacher, F. Fernàndez, A. Fuchs, N. Golnik, V. Lacoste, A. Leuschner, S. Sandri, M. Silari (Editor), F. Spurny, B. Wiegel, P. Wright, "Complex Workplace Radiation Fields at European High-Energy Accelerators and Thermonuclear Fusion Facilities", CERN European Organization For Nuclear Research, Report CERN 2006-007, Geneva, July 24, 2006

[3] Dinter H., Tesch K., Dworak D., "Studies On The Neutron Field Behind Shielding Of Proton Accelerators. Part I: Concrete Shielding". Nuclear Instruments \& Methods. Phys. Res. A 368, 265-272, 1996

[4] Dinter H., Leuschner A., Tesch K., Dworak D., Loskiewicz J. , "Calculation Of Hadron Yields. Around Thick Targets And doses Behind Concrete Shielding Of High Energy Accelerators". Nuclear Instruments \& Methods, Phys. Res. A 455, 460-469, 2000

[5] Method And System For Determining The Flow Of High-Energy Neutrons, DESY, PCT Application WO 2006/097338 A1, March 20, 2006, published Sep. 21, 2006 